\title{
Software-based Versatile Video Coding Decoder Parallelization
}

\author{
Srinivas Gudumasu \\ InterDigital Communications, Inc \\ San Diego, California, USA \\ Srinivas.gudumasu@interdigital.com
}

\author{
Saurav Bandyopadhyay ${ }^{\dagger}$ \\ saurav@ieee.org
}

\author{
Yong $\mathrm{He}^{\dagger}$ \\ eeehey@gmail.com
}

\begin{abstract}
Versatile Video Coding (VVC) standard is currently being prepared as the latest video coding standard of the ITU-T and ISO/IEC. The primary goal of the VVC, expected to be finalized in 2020, is to further improve compression performance compared to its predecessor HEVC. The frame level, slice level or Wavefront parallel processing (WPP) existing in VTM (VVC Test Model) doesn't fully utilize the CPU capabilities available in today's multicore systems. Moreover, VTM decoder sequentially processes the decoding tasks. This design is not parallelization friendly. This paper proposes re-designed decoding tasks that parallelize the decoder using: 1. Load balanced task parallelization and 2. CTU (Coding Tree Unit) based data parallelization. The design overcomes the limitations of the existing parallelization techniques by fully utilizing the available CPU computation resource without compromising on the coding efficiency and the memory bandwidth. The parallelization of CABAC and the slice decoding tasks is based on a load sharing scheme, while parallelization of each sub-module of the slice decoding task uses CTU level data parallelization. The parallelization scheme may either remain restricted within an individual decoding task or utilize between task parallelization. Such parallelization techniques achieve real-time VVC decoding on multi-core CPUs, for bitstreams generated using VTM5.0 using Random-Access configuration. An overall average decoding time reduction of $88.97 \%$ (w.r.t. VTM5.0 decoder) is achieved for $4 \mathrm{~K}$ sequences on a 10 -core processor.
\end{abstract}

\section{CCS CONCEPTS}

-General and reference Cross-computing tools and techniques $\sim$ Design $\bullet$ Computing methodologies $\sim$ Parallel computing methodologies $\sim$ Parallel algorithms $\sim$ Massively parallel algorithms

\section{KEYWORDS}

Versatile Video Coding, parallelization, CTU level parallelization, load sharing based parallelization

${ }^{\dagger}$ Messrs. Bandyopadhyay and He were each employed by InterDigital Communications, Inc. at the time of submission.

Permission to make digital or hard copies of part or all of this work for personal or classroom use is granted without fee provided that copies are not made or distributed for profit or commercial advantage and that copies bear this notice and the full citation on the first page. Copyrights for third-party components of this work must be honored. For all other uses, contact the Owner/Author.

MMSYS'20, June 8-11, 2020, Istanbul, Turkey

(C) 2020 Copyright is held by the owner/author(s).

ACM ISBN 978-1-4503-6845-2/20/06.

https://doi.org/10.1145/3339825.3391871

\section{ACM Reference format:}

Srinivas Gudumasu, Yong He and Saurav Bandyopadhyay. 2020. Softwarebased Versatile Video Coding Decoder Parallelization. In MMSys '20: 11th ACM Multimedia Systems Conference, June 8-11, 2020, Istanbul, Turkey. ACM, New York, NY, USA, 11 pages. https://doi.org/10.1145/3339825.3391871

\section{Introduction}

Joint Video Exploration Team (JVET) was formed in Oct. 2015 by ITU-T VCEG and ISO/IEC MPEG to begin study of advanced technologies that could enable substantial enhancement of coding efficiency. One reference software called joint exploration model (JEM) was maintained by the JVET by integrating several additional coding tools on top of the HEVC [1][2][3][4] test model (HM).

In Oct. 2017, joint call for proposals (CfP) on video compression with capability beyond HEVC was issued by ITU-T and ISO/IEC [5]. In Apr. 2018, 23 CfP responses were received [6] and evaluated at the 10-th JVET meeting, which demonstrated compression efficiency gain of around 40\% over the HEVC [7]. Based on such evaluation results, the JVET launched a new project to develop the new generation video coding standard that is named as Versatile Video Coding. During the same month, a reference software codebase, called VVC test model, was established for demonstrating a reference implementation of the VVC standard [8]. To facilitate the assessment of new coding tools, another reference software base called benchmark set was generated.

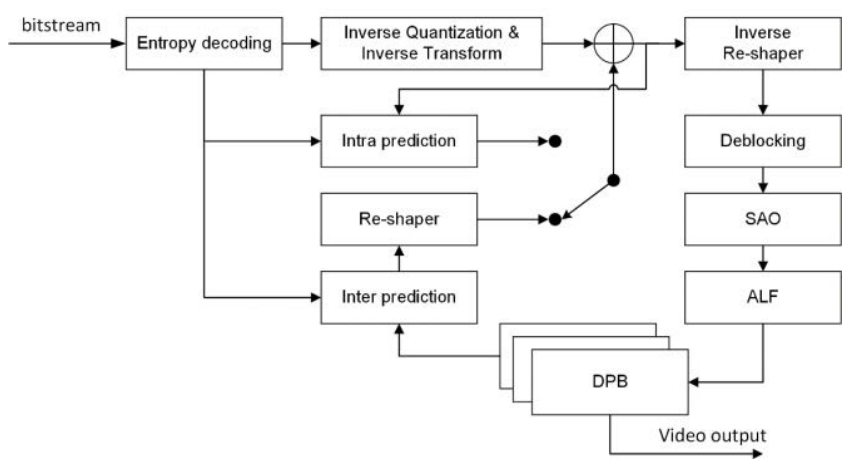

Figure 1: VVC Decoding architecture 
Figure 1 gives a general block diagram of a block-based video decoder. The video bit-stream is first unpacked, and entropy decoded at entropy decoding unit. The coding mode and prediction information are sent to either the spatial prediction unit (if intra coded) or the temporal prediction unit (if inter coded) to form the prediction block. The residual transform coefficients are sent to inverse quantization unit and inverse transform unit to reconstruct the residual block. The prediction block and the residual block are then added together. The reconstructed block may further go through in-loop filtering before it is stored in reference picture buffer. The reconstructed video in reference picture store is then sent out to drive a display device, as well as used to predict future video blocks.

Context-based adaptive binary arithmetic coding (CABAC) is used for entropy coding in VVC. The CABAC scheme in VVC is the same as in HEVC, with two improvements: Multi-hypothesis probability models with different probability updating speeds are employed in the arithmetic coding engine, and the transform coefficients are coded with an optimized context model selection.

Smart mobile devices and mobile internet are taking root in people's daily lives and the demand on video and its quality is very high. Next-generation video codecs such as VVC are required to improve coding efficiency and quality, while legacy codecs enable playback on outdated devices. It is a challenge to achieve real-time decoding with the computationally expensive VVC codec. Live and video on demand playback applications can use such real-time VVC decoding for high quality, smooth playback and better user experience with reduced bandwidth.

This paper addresses the limitations with existing parallelization techniques in VVC decoder and proposes the Multi-threaded (MT) parallel processing framework to fully utilize the available CPU computation resources without compromising on the coding efficiency. The proposed multi-threaded framework uses CTU level parallel processing techniques without compromising on the memory bandwidth. The paper discusses various optimization techniques used to achieve real-time VVC decoding on heterogenous platforms with multi-core CPUs, for those bitstreams generated using the VVC reference encoder with default common test condition configuration. Equivalent techniques proposed for the decoder could also be used with the VVC encoder framework.

The rest of this paper is organized as follows, Section 2 reviews the existing parallelization techniques in video codecs. Section 3 introduces the proposed parallel processing framework, Section 4 provides the details of video player application, Section 5 presents experimental results and Section 6 presents the future work on VVC decoder optimization techniques. Finally, conclusions are drawn in Section 7.

\section{Background and Related work}

Several published articles have exploited the parallelization capability of the video codecs prior to VVC on CPU, GPU or heterogenous platform. In [9], the authors provide a discussion of the compression techniques, existing video standards and various parallelization approaches. In [10], the authors proposed a HEVC de-blocking filter that exploits independent regions of the frame and reduces the overall memory access. In [11] [12], GPU is used to accelerate HEVC decoding tasks of de-quantization, inverse transform, intra prediction, deblocking filter and Sample Adaptive offset (SAO). In [13], a GPU based parallel algorithm is proposed including parallel computation of sample classification, statistics collection for each coding tree block, parallel computation of best offset values and minimum distortion for each class of edge offset and band offset, SAO merging and SAO filtering. An implementation of a VVC software player on mobile devices is shown in [14]. A parallelization scheme for HEVC is proposed in [15][16]. A real-time thread-scheduling mechanism for Scalable HEVC is proposed in [17].

Frame-level parallelism consists of processing multiple frames at the same time while satisfying the motion compensation dependencies. One of the major limitations of the frame level parallelism is that the level of parallelism is determined by the length of the motion vector. This is a major bottleneck, especially for sequences with large motion.

Slice level parallelism allows for processing the slices independently from one another. The major drawback of this level of parallelism is that the number of slices is determined by the encoder. Moreover, in most cases, the codec may be limited to use one slice per frame resulting in almost no slice level parallelism. Additionally, using multiple slices may reduce the coding efficiency.

Above shortcomings of the frame level or slice level parallelism can be overcome by employing Wavefront Parallel Processing (WPP) and Tiles [18]. Both techniques allow subdivision of each picture into multiple partitions that can be processed in parallel. Each partition contains an integer number of coding units that must not have dependencies on other partitions. Usage of tiles reduces the coding efficiency and complicates the raster scan processing. However, the major drawback in tile usage is the rate-distortion loss that would be significant for a higher number of tiles due to the lack of dependency between partitions.

Wavefront parallel processing (WPP) allows for partitioning the picture without breaking the coding dependency or resetting the CABAC probabilities as in parallelism using Slices or Tiles [18]. WPP method partitions one picture into CTU rows and allows prediction and entropy coding across the CTU row boundaries. For this reason, WPP results in lower loss of compression efficiency compared to Slice and Tile based parallel processing methods. However, the Wavefront dependencies do not allow all the CTU 
rows to simultaneously start decoding. Therefore, the rows don't finish the decoding at the same time either. This introduces parallelization inefficiencies that become more evident when a high number of WPP threads are used for decoding.

Overlapped Wavefront (OWF) Parallelism improves the implementation efficiency of WPP by overlapping the execution of consecutive pictures. The dependency in OWF technique is caused by motion search. In OWF, a CTU is not ready for decoding until all its reference pixels within the motion search area in the reference picture have been decoded. The motion search dependency limits the throughput of frame-level parallel processing threads for encoding multiple frames. This problem worsens when the center of the search window (decided by a motion predictor) is located toward the lower part of the reference pictures. On the other hand, restricting the motion vector leads to noticeable coding loss for videos that exhibit fast vertical motion. Therefore, none of the traditional parallelization techniques can fully utilize the CPU processing capabilities available on today's multi-core systems.

\section{Parallel Solution}

\subsection{Overall Parallelization Architecture}

To improve the degree of parallelism of the VVC decoder tasks as in Figure 1, we propose to divide these tasks for each picture into the following sub-tasks.

\section{CABAC decoding}

2. Motion Vector Component Derivation (MVCD)

3. Inter coding unit (CU) \& combined intra and inter picture prediction (CIIP) [6][8] inter reconstruction

4. Intra CU \& CIIP intra reconstruction

5. Inverse reshaping

6. In-loop filtering

7. De-blocking Filter

8. Sample Adaptive Offset

9. Adaptive Loop Filter (ALF)

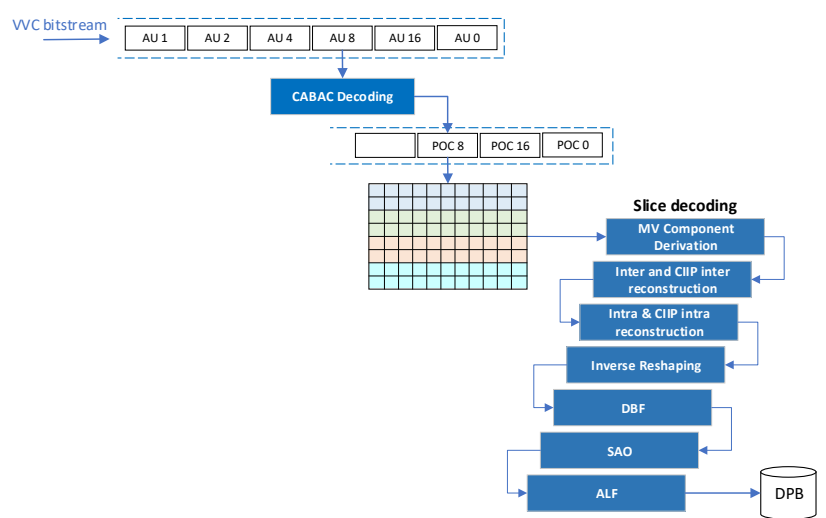

Figure 2: Overview parallelization architecture
The paper proposes a finer granularity of parallelism without compromising on the coding efficiency. The finer granularity of the parallelism is achieved by processing in parallel the CABAC decoding and the rest of the slice decoding stages referred as slice decoding tasks. The architectural diagram of slice/picture decoding tasks is shown in Figure 2. Parallelization of each slice decoding task e.g. Inter, Intra, Re-shaper and In-loop filters is shown in Figure 3 and described in Section 3.2.

The parallelization of VVC decoding tasks can be categorized into the following 2 approaches.

$$
\begin{array}{ll}
\text { I. } & \text { Load balanced task parallelization } \\
\text { II. } & \text { CTU based data parallelization; }
\end{array}
$$

VTM decoder pipeline is divided into CABAC decoding and slice decoding tasks. CABAC decoding and slice decoding are running simultaneously on different slices or pictures due to data dependency. CABAC decoding is usually faster than slice decoding, therefore a single CABAC thread for a frame is generally adequate. In case of very low quality parameter (QP) or more complicated content, single thread CABAC decoding may be slower than the slice decoding. In this case, multiple threads may be allocated to $\mathrm{CABAC}$ decoding to balance the workload between CABAC decoding and slice decoding.

Approach II is applied on slice decoding tasks, including Inter and CIIP inter reconstruction, Intra and CIIP intra reconstruction, motion vector derivation, Re-shaping, De-blocking filter, Sample Adaptive Offset and Adaptive Loop Filtering. Approach II provides fine granularity parallelism on top of existing tile-based parallelization.

For the proposed VVC decoding pipeline design, one thread is reserved for $\mathrm{CABAC}$ decoding and the rest of the threads are allocated for the slice decoding. The CABAC decoded output is stored in a first in first out (FIFO) queue. This thread continues CABAC decoding of the sub-sequent NAL units. Slice decoding threads execute on the CABAC decoded output as in Figure 1.

\subsection{Parallelization of slice decoding tasks}

In VVC, a picture is partitioned into CTUs [20][21]. Such partitioning enables the data parallelization at each slice/picture decoding task.

\subsubsection{Thread scheduling}

In this section, we illustrate the proposed thread scheduling mechanism shown in Figure 3. This uses a strategy similar to the thread scheduling mechanism of [17]. 


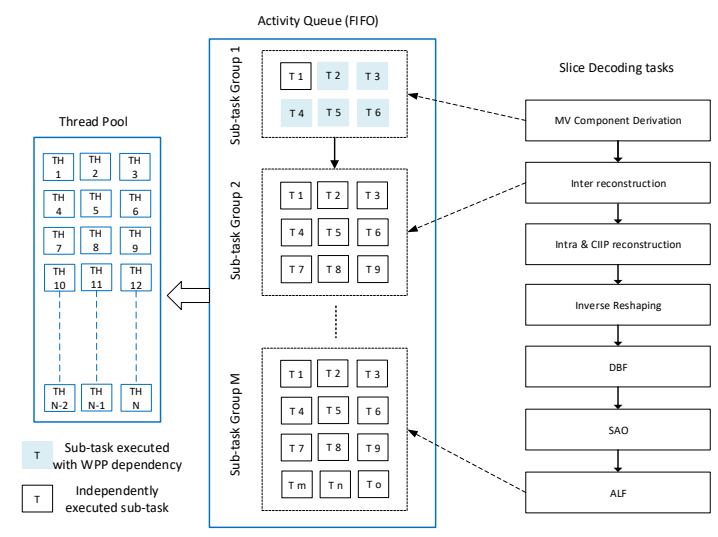

Figure 3: Thread scheduling mechanism for slice/picture decoding tasks

A thread pool and a FIFO activity queue are created for processing the slice decoding task activities. A thread pool is created based on the number of available CPU cores in the multi-core system. For example, a thread pool of 20 threads is created for Intel Core ${ }^{\mathrm{TM}}$ processor with 10 cores. Each slice decoding task shown in Figure 2 is further divided into multiple sub-tasks. Each sub-task may consist of a CTU or a group of CTUs or one row of CTUs. A scheduling thread pushes the sub-tasks into an activity queue. Any free thread from the thread pool fetches the first available sub-task from the FIFO activity queue and executes the task. As a result, the sub-tasks of a decoding task can be executed in parallel without synchronization overhead. All the sub-tasks of one decoding task shall be completed before kicking off the sub-tasks of the next decoding task.

\subsubsection{Motion Vector Component Derivation (MVCD)}

Motion vector component derivation process, as in Figure 4, is completed for the entire inter slice before proceeding to the subsequent slice decoding process. Motion vector component derivation can depend on its left, top, and/or top right neighbor $\mathrm{CU}$ motion vector, which is referred to as WPP dependency. To achieve better parallel processing capabilities, the motion vector component derivation process is divided into sub-tasks. Each sub-task consists of one CTU row of motion vector derivation process.

The scheduling thread pushes the MVCD sub-task to the activity queue in the order of increasing CTU row number. An available thread from the thread pool will fetch the first available CTU row from the activity queue and derive the motion vector of each CTU in sequential order until completion of the entire CTU row. The synchronization overhead is required between threads to guarantee the data dependency. The maximum number of threads that can be used for MVCD parallel processing is equal to the number of CTU rows within a slice.
The proposed parallelization scheme achieves an average reduction of $70.16 \%$ MVCD slice decoding task time.

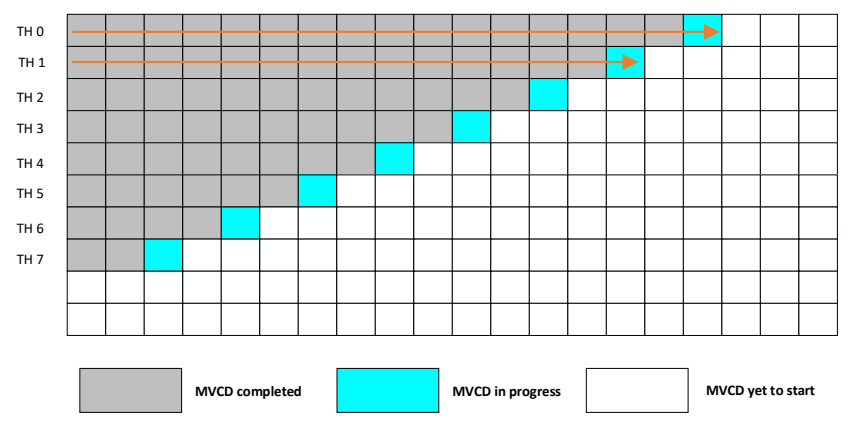

Figure 4: Thread scheduling mechanism for MVCD task

\subsubsection{Inter CU reconstruction}

After motion vector component derivation, inter $\mathrm{CU}$ reconstruction [22][23][24][25] along with the CIIP CU inter reconstruction is processed for the entire picture. The inter $\mathrm{CU}$ reconstruction process is divided into multiple sub-tasks. Each inter reconstruction sub-task may consist of one or a group of CTUs.

The scheduling thread pushes the inter $\mathrm{CU}$ reconstruction process of a CTU or a group of CTUs to the activity queue in the increasing CTU number order. An available thread will fetch the first available CTU or CTU group from the activity queue and perform the inter $\mathrm{CU}$ reconstruction process. All threads perform the inter $\mathrm{CU}$ reconstruction process of the CTU groups in parallel as shown in Figure 5. This way the complexity of the whole slice/picture inter $\mathrm{CU}$ reconstruction can be reduced significantly.

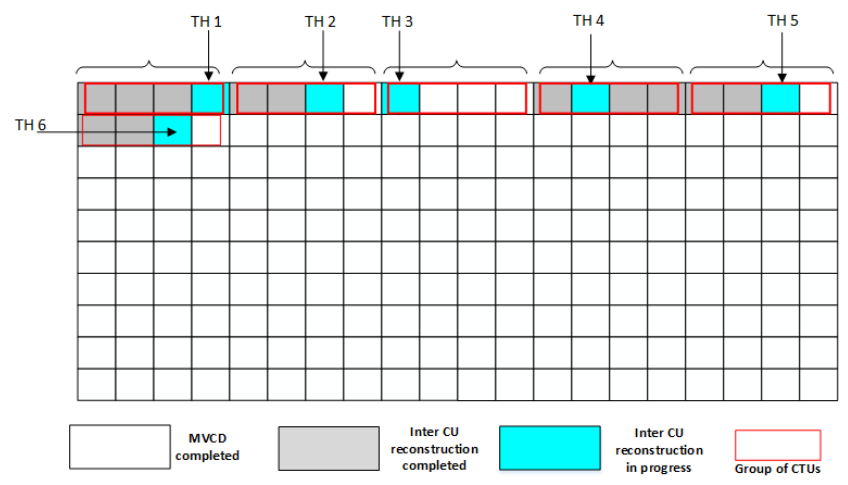

Figure 5: Thread scheduling mechanism for Inter CU reconstruction task

The inter prediction of the CIIP mode CU can be combined with other inter CUs prediction process. Inter CU and CIIP inter CU prediction process can be parallelized across all CTUs of a picture. This way the CIIP inter CUs reconstruction process time can be reduced significantly by processing them in parallel using multiple 
Software-based Versatile Video Coding Decoder Parallelization

slice decoding threads. CIIP intra CUs reconstruction process is combined with other intra CUs reconstruction process.

The proposed parallelization scheme achieves an average reduction of $88.25 \%$ inter CU reconstruction task time.

\subsubsection{Intra CU reconstruction}

A traditional WPP parallelization scheme is applied for the intra CU prediction [22][26][27][28]. In this approach, each CTU row is treated as a sub-task. Each sub-task is processed by a free slice decoding thread as in Figure 6. Multiple CTU rows are executed in parallel with synchronization overhead. Each CTU cannot be processed until its left and top-right neighboring CTUs are available. CIIP CU intra prediction is also processed along with normal intra CUs prediction.

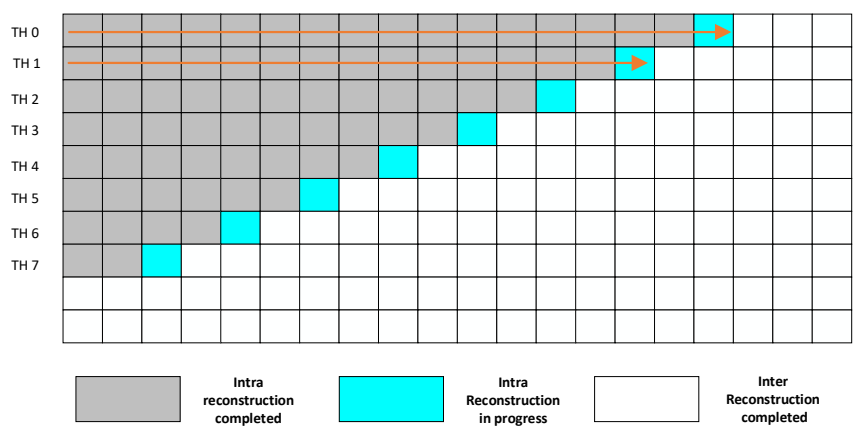

Figure 6: Thread scheduling mechanism for Intra reconstruction task

Any CTU containing only CIIP [29] and inter CUs is processed with different scheduling. This is applicable when the neighboring (left, top left, top and top right) CTUs are coded in inter mode only. The scheduling thread creates a sub-task for the current CTU's CIIP intra reconstruction task and pushes it into the activity queue. A free thread executes the CIIP intra reconstruction independently. As a result, all the above mentioned CTUs CIIP intra reconstruction sub-tasks can be executed in parallel as shown in Figure 7.

The proposed parallelization scheme achieves an average reduction of $68.94 \%$ intra CU reconstruction task time.
MMSys'20, June 8-11, 2020, Istanbul, Turkey

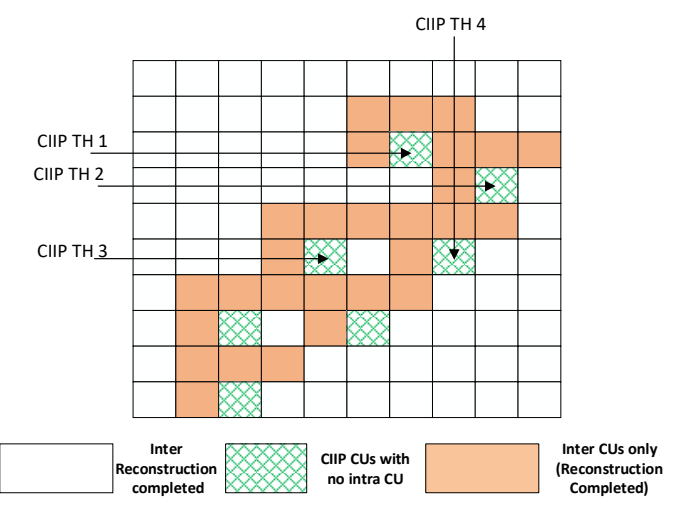

Figure 7: Thread scheduling mechanism for CIIP reconstruction task

\subsubsection{Inverse Re-shaper}

After the $\mathrm{CU}$ reconstruction task, inverse reshaping for the entire picture is executed in parallel using multiple threads. The inverse reshaping process for the entire picture is equally distributed among the available free threads as in Figure 8. For example, when 10 free slice decoding threads are available to process 2160 rows of a $4 \mathrm{~K}$ picture (3840x2160), each slice decoding thread can process inverse reshaping task for 216 rows.

The proposed parallelization scheme achieves an average reduction of $86.79 \%$ inverse re-shaping task time.

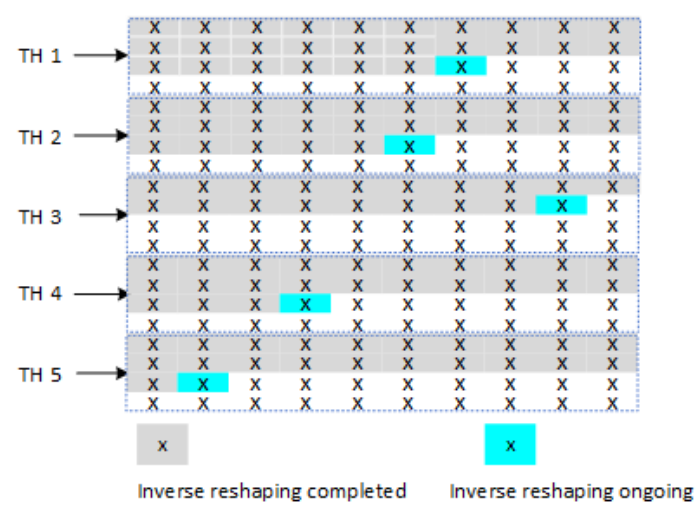

Figure 8: Thread scheduling mechanism for Inverse Reshaping task

\subsubsection{De-blocking filter}

The de-blocking module [22][30][31] in the VVC loop-filtering task is applied immediately after the inverse re-shaper. In VVC, the vertical de-blocking filter is applied on the horizontal de-blocking filter output. Since there is no data dependency between CTUs for either the horizontal or vertical filtering tasks, all CTUs for the whole frame can be processed in parallel as shown in Figure 9. 

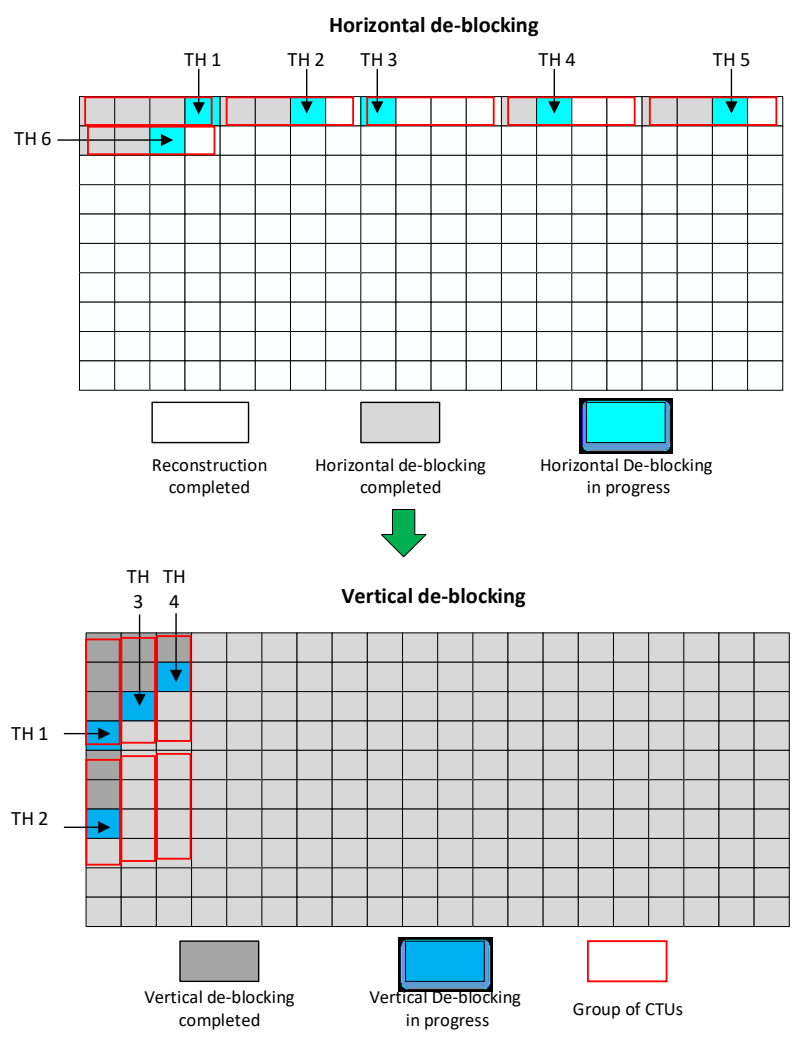

Figure 9: Thread Scheduling mechanism for De-blocking filter task

The first available free thread fetches the next available CTU or CTU group (e.g. 4 CTUs) from the activity queue for horizontal deblocking filtering. Another free thread will fetch the next available CTU or CTU group from the activity queue and will start processing horizontal DBF in parallel with other slice decoding thread(s). This way, all CTUs in the entire frame can be processed in parallel. While performing vertical de-blocking filtering a condition checks the completion of the horizontal filtering for the current and CTU below. The proposed parallelization scheme achieves an average reduction of $90.22 \%$ for the de-blocking filtering task time.

\subsubsection{Sample Adaptive Offset (SAO) \& Adaptive Loop Filter (ALF)}

In VTM5.0, SAO [32] and ALF are applied after the de-blocking filter. We propose to process all CTUs for the whole frame in parallel for either ALF or SAO. ALF process is divided into multiple sub-tasks with each task containing one or a group of CTUs. The scheduling thread pushes one or a group of CTUs subtasks to the activity queue. Free threads will fetch the first available sub-task and execute ALF process on those CTU(s). This way ALF process is completely processed in parallel for the entire picture. Following the completion of the ALF process, the same thread scheduling mechanism is applied for SAO processing.
The proposed parallelization scheme achieves an average reduction of $88.17 \%$ and $72.28 \%$ for ALF and SAO task time respectively.

\section{Video Player}

To illustrate the proposed parallel decoder framework, we implemented a VVC video player application using the OpenGL framework. The player application fetches the decoded output from the decoded picture buffer (DPB) and renders it to the output device in real-time. OpenGL based pixel shader is used to convert the 10 bit 4:2:0 YUV format video frame to RGB32 format in real-time for display purpose.

\section{Experimental Results}

The following experiments were conducted using the VTM-5.0 reference software [33] and the JVET common test conditions [34] to collect the decoding time of the proposed techniques in the Random-Access configuration. An Intel Core I-9-9900 X-series processor with 10 cores running at $3.5 \mathrm{GHz}$ is used to generate experimental results for both the VTM5.0 anchor and for the proposed parallel decoder implementation. Intel CPUs with hyper threading technology enabled can support 2 logical processors per core. To fully utilize the CPU resources, 20 decoding threads are used in our experiments on 10 core I-9-9900 machine.

The test results of the proposed implementation are compared against the VTM5.0 anchor. The decoding time reduction is calculated as the percentage saving from the proposed method(s) over VTM5.0. Mathematically, it can be represented as

$$
\text { Time Saving }(\%)=\frac{\left(T_{V T M 5.0}-T_{\text {Proposed }}\right)}{T_{V T M 5.0}} \times 100
$$

where, $T_{V T M 5.0}$ is the time taken by the individual modules of default VTM5.0 and $T_{\text {Proposed }}$ is the corresponding module time from the proposed method.

Five 4K (CatRobot1, DaylightRoad2, CampFireParty, Tango2 and FoodMarket4) and five 1080P (BQTerrace, RitualDance, MarketPlace, BasketballDrive and Cactus) sequences defined in the JVET common test conditions [34] are used for our experiments. Test bitstreams are generated from Class A: 3840x2160 10 bit 4:2:0 YUV and Class B: 1920x1080 8 bit 4:2:0 YUV format sequences at QPs: 22, 27, 32, 37. More details about the availability of common test condition sequences are provided in [34].

\subsection{Slice decoding performance}

The percentage decoding time reduction due to parallelization is calculated based on (1) for each task of slice decoding. The test results of all the proposed methods using 20 decoding threads are compared against the VTM5.0 anchor in this section. Figure 10 
Software-based Versatile Video Coding Decoder Parallelization

shows the percentage contribution of slice decoding tasks based on VTM5.0 and Figure 11 is based on the proposed method at QP=37 for the $4 \mathrm{~K}$ sequences CatRobot1, DaylightRoad2 and CampFireParty.
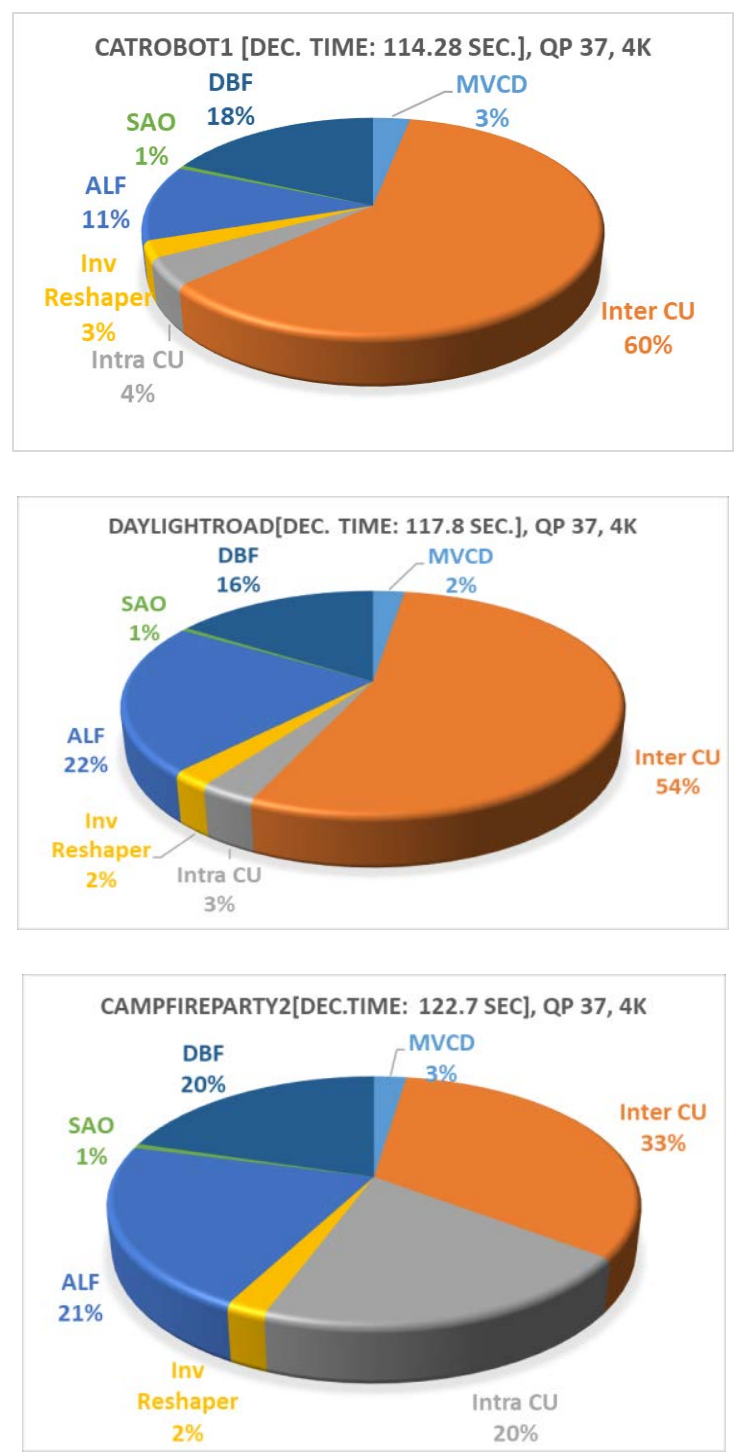

Figure 10: VTM5.0 complexity analysis at QP: 37

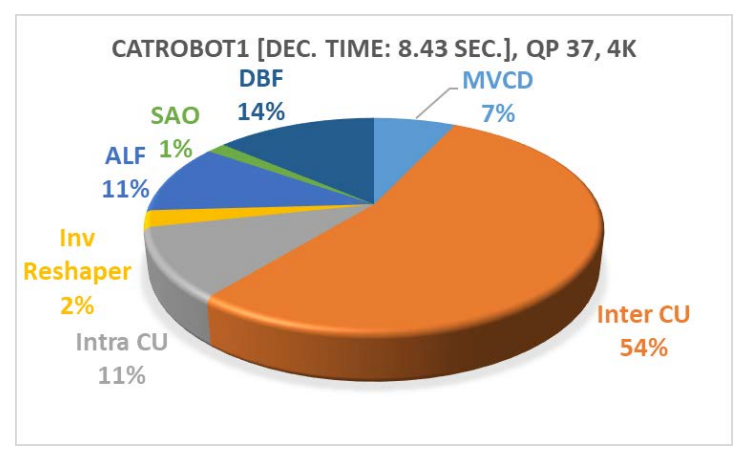

MMSys'20, June 8-11, 2020, Istanbul, Turkey
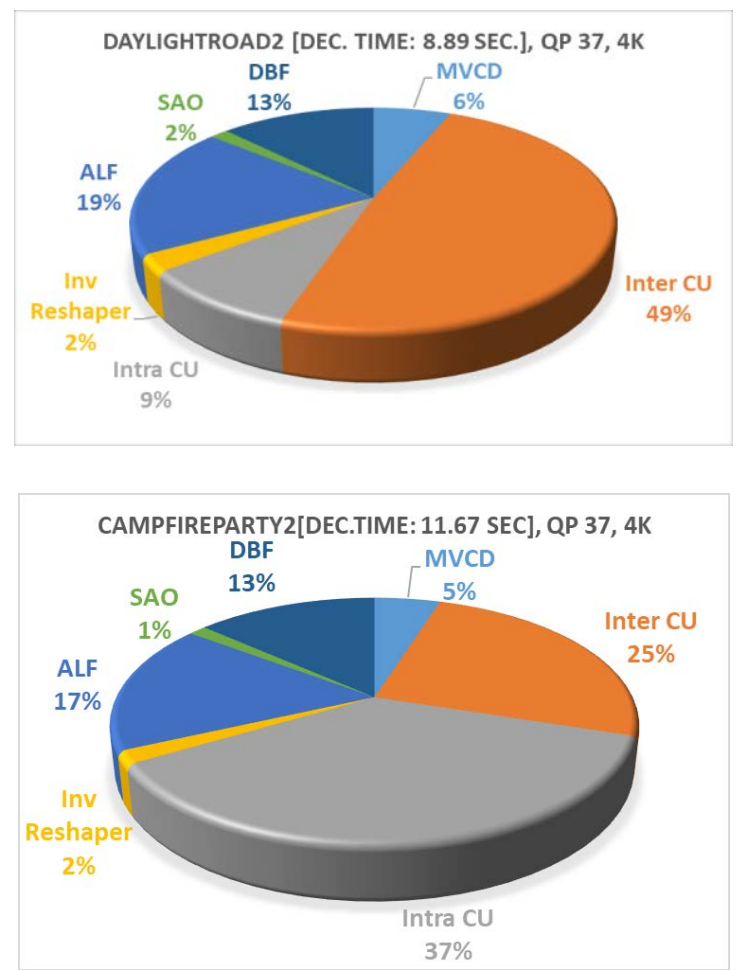

Figure 11: Proposed solution complexity analysis at QP: 37

Table 1 to Table 7 shows the processing time comparison details between the parallel solution and VTM5.0. The experiments are conducted for inter prediction, deblocking filter, SAO and DBF tasks using one CTU in the CTU group.

Table 1: MVCD decoding time

\begin{tabular}{|c|c|c|c|c|c|}
\hline \multirow[t]{2}{*}{ Sequences } & \multirow[t]{2}{*}{ QP } & \multicolumn{4}{|c|}{ Decoding time, $\%$ improvement for MVCD } \\
\hline & & $\begin{array}{l}\text { VTM5.0 } \\
(\mathrm{sec})\end{array}$ & $\begin{array}{l}\text { Proposed } \\
(\mathrm{sec})\end{array}$ & $\begin{array}{l}\text { Time } \\
\text { reduction } \\
(\%)\end{array}$ & $\begin{array}{l}\text { Average } \\
\text { reduction (\%) }\end{array}$ \\
\hline \multirow[t]{4}{*}{ CatRobot1 } & 22 & 3.886 & 1.076 & 72.31 & \multirow[t]{4}{*}{73.2} \\
\hline & 27 & 2.582 & 0.662 & 74.36 & \\
\hline & 32 & 2.136 & 0.551 & 74.20 & \\
\hline & 37 & 1.848 & 0.519 & 71.92 & \\
\hline \multirow[t]{4}{*}{ DaylightRoad2 } & 22 & 4.452 & 1.334 & 70.04 & \multirow[t]{4}{*}{71.09} \\
\hline & 27 & 2.385 & 0.639 & 73.21 & \\
\hline & 32 & 1.867 & 0.54 & 71.08 & \\
\hline & 37 & 1.633 & 0.489 & 70.06 & \\
\hline \multirow[t]{4}{*}{ CampFireParty } & 22 & 4.589 & 1.865 & 59.36 & \multirow[t]{4}{*}{66.21} \\
\hline & 27 & 2.326 & 0.728 & 68.70 & \\
\hline & 32 & 1.936 & 0.619 & 68.03 & \\
\hline & 37 & 1.604 & 0.501 & 68.77 & \\
\hline
\end{tabular}


MMSys'20, June 8-11, 2020, Istanbul, Turkey

Table 2: Inter CU reconstruction decoding time

\begin{tabular}{|c|c|c|c|c|c|}
\hline \multirow[t]{2}{*}{ Sequences } & \multirow[t]{2}{*}{ QP } & \multicolumn{4}{|c|}{$\begin{array}{l}\text { Decoding time, \% improvement for Inter } \mathrm{CU} \\
\text { reconstruction }\end{array}$} \\
\hline & & $\begin{array}{l}\text { VTM5.0 } \\
\text { (sec) }\end{array}$ & $\begin{array}{l}\text { Proposed } \\
(\mathrm{sec})\end{array}$ & $\begin{array}{l}\text { Time } \\
\text { reduction } \\
(\%)\end{array}$ & $\begin{array}{l}\text { Average } \\
\text { reduction } \\
(\%)\end{array}$ \\
\hline \multirow[t]{4}{*}{ CatRobot1 } & 22 & 39.395 & 4.498 & 88.58 & \multirow[t]{4}{*}{88.97} \\
\hline & 27 & 37.887 & 4.12 & 89.13 & \\
\hline & 32 & 36.822 & 3.881 & 89.46 & \\
\hline & 37 & 35.469 & 4.009 & 88.70 & \\
\hline \multirow[t]{4}{*}{ DaylightRoad2 } & 22 & 35.872 & 4.239 & 88.18 & \multirow[t]{4}{*}{88.99} \\
\hline & 27 & 38.602 & 4.116 & 89.34 & \\
\hline & 32 & 37.255 & 4.07 & 89.08 & \\
\hline & 37 & 36.142 & 3.841 & 89.37 & \\
\hline \multirow[t]{4}{*}{ CampFireParty } & 22 & 23.464 & 4.129 & 82.40 & \multirow[t]{4}{*}{86.79} \\
\hline & 27 & 23.013 & 2.696 & 88.28 & \\
\hline & 32 & 22.471 & 2.686 & 88.05 & \\
\hline & 37 & 22.268 & 2.581 & 88.41 & \\
\hline
\end{tabular}

Table 3: Intra CU reconstruction decoding time

\begin{tabular}{|c|c|c|c|c|c|}
\hline \multirow[t]{2}{*}{ Sequences } & \multirow[t]{2}{*}{ QP } & \multicolumn{4}{|c|}{ Decoding time, $\%$ improvement for Intra CU reconstruction } \\
\hline & & $\begin{array}{l}\text { VTM5.0 } \\
(\mathrm{sec})\end{array}$ & $\begin{array}{l}\text { Proposed } \\
(\mathrm{sec})\end{array}$ & $\begin{array}{l}\text { Time } \\
\text { reduction } \\
(\%)\end{array}$ & $\begin{array}{l}\text { Average } \\
\text { reduction (\%) }\end{array}$ \\
\hline \multirow[t]{4}{*}{ CatRobot1 } & 22 & 8.236 & 2.487 & 69.80 & \multirow[t]{4}{*}{67.60} \\
\hline & 27 & 4.242 & 1.367 & 67.77 & \\
\hline & 32 & 3.046 & 1.027 & 66.28 & \\
\hline & 37 & 2.367 & 0.792 & 66.54 & \\
\hline \multirow[t]{4}{*}{ DaylightRoad2 } & 22 & 15.539 & 4.387 & 71.77 & \multirow[t]{4}{*}{67.73} \\
\hline & 27 & 5.132 & 1.613 & 68.57 & \\
\hline & 32 & 3.098 & 1.051 & 66.07 & \\
\hline & 37 & 2.070 & 0.735 & 64.49 & \\
\hline \multirow[t]{4}{*}{ CampFireParty } & 22 & 39.245 & 11.498 & 70.70 & \multirow[t]{4}{*}{71.49} \\
\hline & 27 & 21.908 & 6.395 & 70.81 & \\
\hline & 32 & 16.606 & 4.696 & 71.72 & \\
\hline & 37 & 13.735 & 3.748 & 72.71 & \\
\hline
\end{tabular}

Table 4: Inverse Re-shaper decoding time

\begin{tabular}{|c|c|c|c|c|c|}
\hline \multirow[t]{2}{*}{ Sequences } & \multirow[t]{2}{*}{ QP } & \multicolumn{4}{|c|}{ Decoding time, $\%$ improvement for Inverse Re-Shaper } \\
\hline & & $\begin{array}{l}\text { VTM5.0 } \\
\text { (sec) }\end{array}$ & $\begin{array}{l}\text { Proposed } \\
(\mathrm{sec})\end{array}$ & $\begin{array}{l}\text { Time } \\
\text { reduction } \\
(\%)\end{array}$ & $\begin{array}{l}\text { Average } \\
\text { reduction (\%) }\end{array}$ \\
\hline \multirow[t]{3}{*}{ CatRobot1 } & 27 & 1.465 & 0.184 & 87.44 & \multirow[t]{3}{*}{87.22} \\
\hline & 32 & 1.466 & 0.191 & 86.97 & \\
\hline & 37 & 1.468 & 0.187 & 87.26 & \\
\hline \multirow[t]{3}{*}{ DaylightRoad2 } & 27 & 1.463 & 0.2 & 86.33 & \multirow[t]{3}{*}{86.42} \\
\hline & 32 & 1.463 & 0.205 & 85.99 & \\
\hline & 37 & 1.464 & 0.191 & 86.95 & \\
\hline \multirow[t]{3}{*}{ CampFireParty } & 27 & 1.451 & 0.202 & 86.08 & \multirow[t]{3}{*}{86.74} \\
\hline & 32 & 1.447 & 0.199 & 86.25 & \\
\hline & 37 & 1.436 & 0.174 & 87.88 & \\
\hline
\end{tabular}

Table 5: Adaptive Loop Filter decoding time

\begin{tabular}{|c|c|c|c|c|c|}
\hline \multirow[t]{2}{*}{ Sequences } & \multirow[t]{2}{*}{ QP } & \multicolumn{4}{|c|}{ Decoding time, \% improvement for Adaptive Loop Filter } \\
\hline & & $\begin{array}{l}\begin{array}{l}\text { VTM5.0 } \\
(\mathrm{sec})\end{array}\end{array}$ & $\begin{array}{l}\text { Proposed } \\
(\mathrm{sec})\end{array}$ & $\begin{array}{l}\text { Time } \\
\text { reduction } \\
(\%) \\
\end{array}$ & $\begin{array}{l}\text { Average } \\
\text { reduction (\%) }\end{array}$ \\
\hline \multirow[t]{4}{*}{ CatRobot1 } & 22 & 19.569 & 2.3 & 88.25 & \multirow[t]{4}{*}{88.55} \\
\hline & 27 & 17.502 & 2.044 & 88.32 & \\
\hline & 32 & 15.071 & 1.582 & 89.50 & \\
\hline & 37 & 6.664 & 0.79 & 88.15 & \\
\hline \multirow[t]{4}{*}{ DaylightRoad2 } & 22 & 19.620 & 2.292 & 88.32 & \multirow[t]{4}{*}{88.15} \\
\hline & 27 & 19.039 & 2.253 & 88.17 & \\
\hline & 32 & 17.966 & 2.158 & 87.99 & \\
\hline & 37 & 12.619 & 1.5 & 88.11 & \\
\hline \multirow[t]{4}{*}{ CampFireParty } & 22 & 17.677 & 2.111 & 88.06 & \multirow[t]{4}{*}{87.82} \\
\hline & 27 & 16.238 & 1.997 & 87.70 & \\
\hline & 32 & 14.635 & 1.799 & 87.71 & \\
\hline & 37 & 14.440 & 1.761 & 87.80 & \\
\hline
\end{tabular}

S. Gudumasu, S. Bandyopadhyay and Y. He

Table 6: Sample Adaptive Offset decoding time

\begin{tabular}{|c|c|c|c|c|c|}
\hline \multirow[t]{2}{*}{ Sequences } & \multirow[t]{2}{*}{$\mathrm{QP}$} & \multicolumn{4}{|c|}{ Decoding time, $\%$ improvement for Sample Adaptive Offset } \\
\hline & & $\begin{array}{l}\text { VTM5.0 } \\
(\mathrm{sec})\end{array}$ & $\begin{array}{l}\text { Proposed } \\
(\mathrm{sec})\end{array}$ & $\begin{array}{l}\text { Time } \\
\text { reduction } \\
(\%) \\
\end{array}$ & $\begin{array}{l}\text { Average } \\
\text { reduction (\%) }\end{array}$ \\
\hline \multirow[t]{4}{*}{ CatRobot1 } & 22 & 8.557 & 1.741 & 79.65 & \multirow[t]{4}{*}{70.72} \\
\hline & 27 & 1.530 & 0.423 & 72.35 & \\
\hline & 32 & 0.564 & 0.189 & 66.49 & \\
\hline & 37 & 0.334 & 0.119 & 64.37 & \\
\hline \multirow[t]{4}{*}{ DaylightRoad2 } & 22 & 9.011 & 1.791 & 80.12 & \multirow[t]{4}{*}{72.84} \\
\hline & 27 & 2.462 & 0.617 & 74.94 & \\
\hline & 32 & 0.756 & 0.215 & 71.56 & \\
\hline & 37 & 0.326 & 0.115 & 64.72 & \\
\hline \multirow[t]{4}{*}{ CampFireParty } & 22 & 9.148 & 1.976 & 78.40 & \multirow[t]{4}{*}{73.29} \\
\hline & 27 & 4.393 & 1.022 & 76.74 & \\
\hline & 32 & 0.923 & 0.268 & 70.96 & \\
\hline & 37 & 0.407 & 0.134 & 67.08 & \\
\hline
\end{tabular}

Table 7: De-blocking Filter decoding time

\begin{tabular}{|c|c|c|c|c|c|}
\hline \multirow[t]{2}{*}{ Sequences } & \multirow[t]{2}{*}{ QP } & \multicolumn{4}{|c|}{ Decoding time, \% improvement for De-blocking Filter } \\
\hline & & $\begin{array}{l}\begin{array}{l}\text { VTM5.0 } \\
(\mathrm{sec})\end{array}\end{array}$ & $\begin{array}{l}\text { Proposed } \\
(\mathrm{sec})\end{array}$ & $\begin{array}{l}\text { Time } \\
\text { reduction } \\
(\%)\end{array}$ & $\begin{array}{l}\text { Average } \\
\text { reduction (\%) }\end{array}$ \\
\hline \multirow[t]{4}{*}{ CatRobot1 } & 22 & 19.319 & 1.85 & 90.42 & \multirow[t]{4}{*}{90.56} \\
\hline & 27 & 13.974 & 1.322 & 90.54 & \\
\hline & 32 & 12.168 & 1.122 & 90.78 & \\
\hline & 37 & 10.708 & 1.018 & 90.49 & \\
\hline \multirow[t]{4}{*}{ DaylightRoad2 } & 22 & 23.680 & 2.269 & 90.42 & \multirow[t]{4}{*}{90.44} \\
\hline & 27 & 14.679 & 1.416 & 90.35 & \\
\hline & 32 & 12.020 & 1.172 & 90.25 & \\
\hline & 37 & 10.516 & 0.976 & 90.72 & \\
\hline \multirow[t]{4}{*}{ CampFireParty } & 22 & 28.814 & 3.02 & 89.52 & \multirow[t]{4}{*}{89.66} \\
\hline & 27 & 18.978 & 2.015 & 89.38 & \\
\hline & 32 & 16.021 & 1.638 & 89.78 & \\
\hline & 37 & 13.717 & 1.378 & 89.95 & \\
\hline
\end{tabular}

\subsection{Performance improvement combining all decoding tasks}

The total decoding time in frames per second (FPS) achieved using 20 threads by combining Approaches I and II discussed in Section 3 , is reported in Table 8 and Table 9. Five class A and five class B sequences are used for our experiments.

Table 8: FPS improvement combining all decoding tasks for class A sequences

\begin{tabular}{|c|c|c|c|c|}
\hline \multirow[t]{2}{*}{ Sequences } & \multirow[t]{2}{*}{ QP } & \multicolumn{3}{|c|}{ FPS } \\
\hline & & $\begin{array}{l}\text { VTM5.0 } \\
\text { (fps) }\end{array}$ & $\begin{array}{l}\text { Proposed } \\
\text { (fps) }\end{array}$ & $\begin{array}{l}\begin{array}{l}\text { Average } \\
\text { (fps) }\end{array}\end{array}$ \\
\hline \multirow[t]{4}{*}{ CatRobot1 } & 22 & 1.63 & 14.59 & \multirow[t]{4}{*}{26.23} \\
\hline & 27 & 2.10 & 24.54 & \\
\hline & 32 & 2.36 & 30.20 & \\
\hline & 37 & 2.63 & 35.59 & \\
\hline \multirow[t]{4}{*}{ DaylightRoad2 } & 22 & 1.50 & 9.79 & \multirow[t]{4}{*}{23.30} \\
\hline & 27 & 2.03 & 22.28 & \\
\hline & 32 & 2.29 & 27.42 & \\
\hline & 37 & 2.55 & 33.73 & \\
\hline \multirow[t]{4}{*}{ CampFireParty } & 22 & 1.31 & 6.65 & \multirow[t]{4}{*}{17.12} \\
\hline & 27 & 1.82 & 14.77 & \\
\hline & 32 & 2.23 & 21.38 & \\
\hline & 37 & 2.45 & 25.70 & \\
\hline \multirow[t]{4}{*}{ Tango2 } & 22 & 2.64 & 15.18 & \multirow[t]{4}{*}{22.96} \\
\hline & 27 & 3.34 & 24.79 & \\
\hline & 32 & 3.68 & 28.91 & \\
\hline & 37 & 4.04 & 31.87 & \\
\hline \multirow[t]{4}{*}{ FoodMarket4 } & 22 & 2.93 & 21.18 & \multirow[t]{4}{*}{26.84} \\
\hline & 27 & 3.26 & 25.12 & \\
\hline & 32 & 3.70 & 28.37 & \\
\hline & 37 & 4.24 & 32.69 & \\
\hline
\end{tabular}


Table 9: FPS improvement combining all decoding tasks for class B sequences

\begin{tabular}{|c|c|c|c|c|}
\hline \multirow[t]{2}{*}{ Sequences } & \multirow[t]{2}{*}{ QP } & \multicolumn{3}{|c|}{ FPS } \\
\hline & & $\begin{array}{l}\text { VTM5.0 } \\
\text { (fps) }\end{array}$ & $\begin{array}{l}\text { Proposed } \\
\text { (fps) }\end{array}$ & $\begin{array}{l}\text { Average } \\
\text { (fps) }\end{array}$ \\
\hline \multirow[t]{4}{*}{ BQTerrace } & 22 & 6.22 & 30.97 & \multirow[t]{4}{*}{78.54} \\
\hline & 27 & 13.29 & 65.18 & \\
\hline & 32 & 17.48 & 98.43 & \\
\hline & 37 & 20.20 & 119.59 & \\
\hline \multirow[t]{4}{*}{ RitualDance } & 22 & 10.56 & 53.77 & \multirow[t]{4}{*}{75.41} \\
\hline & 27 & 12.83 & 68.34 & \\
\hline & 32 & 14.94 & 82.02 & \\
\hline & 37 & 17.16 & 97.50 & \\
\hline \multirow[t]{4}{*}{ MarketPlace } & 22 & 9.56 & 43.72 & \multirow[t]{4}{*}{79.60} \\
\hline & 27 & 12.51 & 70.29 & \\
\hline & 32 & 15.07 & 91.19 & \\
\hline & 37 & 18.14 & 113.21 & \\
\hline \multirow[t]{4}{*}{ BasketballDrive } & 22 & 10.42 & 35.67 & \multirow[t]{4}{*}{67.59} \\
\hline & 27 & 14.18 & 62.45 & \\
\hline & 32 & 16.58 & 78.32 & \\
\hline & 37 & 18.71 & 93.93 & \\
\hline \multirow[t]{4}{*}{ Cactus } & 22 & 11.16 & 43.78 & \multirow[t]{4}{*}{66.47} \\
\hline & 27 & 17.09 & 64.26 & \\
\hline & 32 & 20.78 & 75.52 & \\
\hline & 37 & 24.39 & 82.32 & \\
\hline
\end{tabular}

The performance improvement in frames per second for all class A bitstreams is shown in Figure 12 and class B bitstreams is shown in Figure 13.

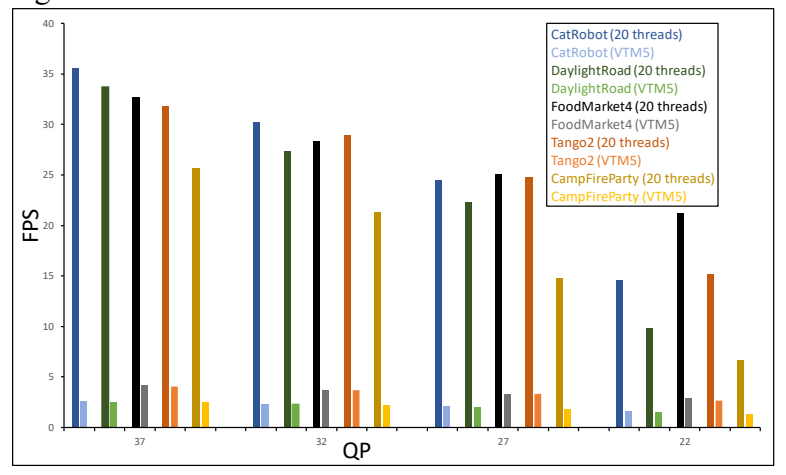

Figure 12: Performance improvement combining all decoding tasks for class A sequences

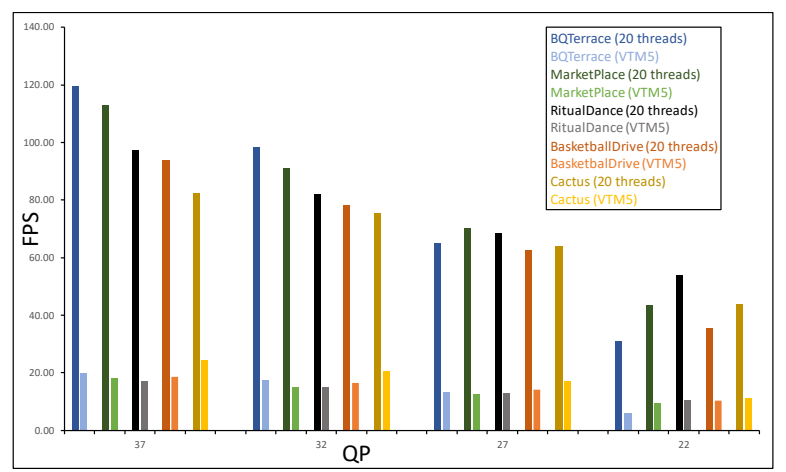

Figure 13: Performance improvement combining all decoding tasks for class B sequences

The average percentage decoding time saving calculated based on (1) with the parallel solution using 20 decoding threads over
VTM5.0 reference software is presented in Table 10. Table 10 reports the data of five class A and five class B sequences for each decoding task discussed above. For each sequence the average decoding time improvement is computed from QPs: 22, 27, 32, 37 bitstreams.

The performance improvement in inter reconstruction, inverse reshaper, deblocking filter and adaptive loop filter is more than $85 \%$ as there is no dependency between CTUs in executing each individual task. The performance improvement in motion vector component derivation and intra reconstruction task is around 50 to $70 \%$ as both tasks are WPP dependent as discussed in section 3.2.2 and 3.2.4.

Table 10: Decoding time improvement for all decoding tasks

\begin{tabular}{|c|c|c|c|c|c|c|c|}
\hline & \multicolumn{6}{|c|}{ Average decoding time, \% improvement for each task using 20 } \\
\hline threads \\
\hline Sequence & $\begin{array}{c}\text { MVC } \\
\text { D }\end{array}$ & Inter & Intra & $\begin{array}{c}\text { Inverse } \\
\text { Reshaper }\end{array}$ & DBF & SAO & ALF \\
\hline CatRobot1 & 73.2 & 88.97 & 67.6 & 87.22 & 90.56 & 70.72 & 88.55 \\
\hline DaylightRoad2 & 71.09 & 88.99 & 67.73 & 86.42 & 90.44 & 72.84 & 88.15 \\
\hline Tango2 & 77.57 & 89.28 & 70.83 & 88.36 & 89.73 & 64.02 & 88.17 \\
\hline FoodMarket4 & 75.92 & 89.13 & 67.43 & 87.33 & 90.07 & 66.81 & 88.02 \\
\hline CampFireParty & 66.21 & 86.79 & 71.49 & 86.74 & 89.66 & 73.29 & 87.82 \\
\hline BQTerrace & 61.40 & 88.05 & 50.30 & 86.48 & 86.56 & 72.70 & 86.49 \\
\hline RitualDance & 58.49 & 88.25 & 57.02 & 83.36 & 88.10 & 66.79 & 86.27 \\
\hline MarketPlace & 59.79 & 88.51 & 56.53 & 85.11 & 87.88 & 66.51 & 86.39 \\
\hline BasketBallDrive & 57.16 & 88.34 & 56.17 & 84.91 & 87.75 & 69.11 & 86.88 \\
\hline Cactus & 56.19 & 87.39 & 48.92 & NA & 86.87 & 67.51 & 84.01 \\
\hline
\end{tabular}

Table 11 provides the CPU and memory usage for VTM5 reference software and the parallel processing approach. The proposed parallel processing approach can effectively use $80-90 \%$ of CPU resources to achieve real-time decoding speed for class A and class B sequences. The memory usage has been increased in the proposed approach to support parallel processing.

Table 11: CPU and memory usage comparison

\begin{tabular}{|c|c|c|c|c|}
\hline & \multicolumn{2}{|c|}{ Class A } & \multicolumn{2}{c|}{ Class B } \\
\hline & $\begin{array}{c}\text { VTM5.0 } \\
\text { Reference }\end{array}$ & Proposed & $\begin{array}{c}\text { VTM5.0 } \\
\text { Reference }\end{array}$ & Proposed \\
\hline CPU (\%) & 7 & 90 & 7 & 80 \\
\hline Memory (MB) & 580 & 890 & 180 & 300 \\
\hline
\end{tabular}

\subsection{Performance improvement w.r.t. number of threads}

The decoding time reduction is dependent on the total number of threads. An Intel Core I-9-9900 X-series processor with 10 physical cores (hyper-threading enabled), was used for our experimentation. Table 12 shows the decoding time w.r.t. number of threads. The table below provides details for three $4 \mathrm{~K}$ sequences from Class A: 3840x2160 10 bit 4:2:0 YUV format at QPs: 22, 27, 32, 37. The corresponding performance variation w.r.t. number of threads is shown in Figure 14. 
Table 12: Performance improvement w.r.t. number of threads

\begin{tabular}{|l|l|l|l|l|l|l|}
\hline Sequences & QP & \multicolumn{5}{|c|}{ Net decoding time (seconds) w.r.t. number of threads } \\
\cline { 3 - 7 } & & VTM5.0 & 4 & 8 & 10 & 20 \\
\hline CatRobot1 & 22 & 1.63 & 8.46 & 13.66 & 14.47 & 14.59 \\
\cline { 2 - 7 } & 27 & 2.10 & 10.06 & 20.32 & 22.49 & 24.54 \\
\cline { 2 - 7 } & 32 & 2.36 & 11.84 & 23.75 & 27.33 & 30.20 \\
\cline { 2 - 7 } & 37 & 2.63 & 14.89 & 29.18 & 32.64 & 35.59 \\
\hline DaylightRoad2 & 22 & 1.50 & 6.96 & 9.96 & 10.38 & 10.32 \\
\cline { 2 - 7 } & 27 & 2.03 & 9.78 & 18.52 & 20.23 & 22.28 \\
\cline { 2 - 7 } & 32 & 2.29 & 10.95 & 22.93 & 25.10 & 27.42 \\
\cline { 2 - 7 } & 37 & 2.55 & 12.41 & 26.71 & 30.21 & 33.73 \\
\hline CampFireParty & 22 & 1.31 & 7.09 & 7.47 & 7.44 & 6.65 \\
\cline { 2 - 7 } & 27 & 1.82 & 8.87 & 14.30 & 14.94 & 15.13 \\
\cline { 2 - 7 } & 32 & 2.23 & 10.07 & 18.97 & 20.13 & 21.38 \\
\cline { 2 - 7 } & 37 & 2.45 & 11.03 & 21.97 & 23.75 & 25.70 \\
\hline
\end{tabular}

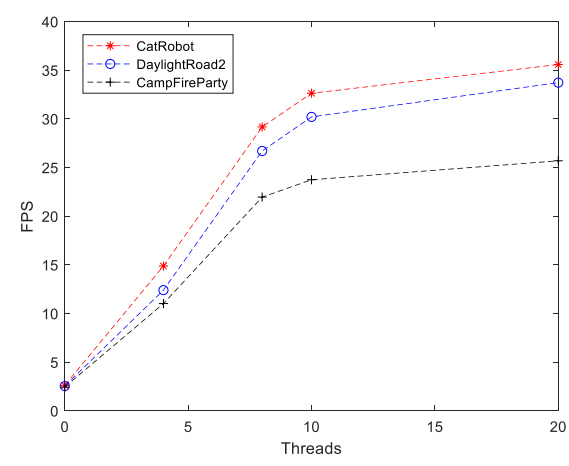

Figure 14: Performance improvement w.r.t. threads

\section{Future Work}

The following steps could be taken to improve the finer granularity parallelism of the pipeline multistage VVC decoder:

1. In the intra CU reconstruction task all the disjoint / independent intra CTUs (CTU with all its intra coded CUs reference pixels coded in inter mode) can be reconstructed in parallel once their referenced pixels inter $\mathrm{CU}$ (left, top and top-right blocks) reconstruction process is completed.

2. Wavefront Parallel Processing (WPP) enabled CABAC decoding can be executed in parallel with the slice decoding tasks. The number of initially allocated threads for CABAC decoding of a picture varies dynamically based on the CABAC performance of the previous pictures.

3. Multiple tasks of the decoder pipeline can be executed in parallel within and across pictures e.g. MVCD, inter $\mathrm{CU}$ reconstruction and intra $\mathrm{CU}$ reconstruction slice decoding tasks can be executed in parallel within a picture or across pictures.

4. WPP parallelization at MVCD and intra CU reconstruction tasks can be improved with preanalysis to find the location of first dependent $\mathrm{CU}$ in each CTU.
5. In order to avoid scanning of the picture multiple times, horizontal and vertical de-blocking filtering can be interleaved. The workload amongst threads can be evenly distributed based on the number of horizontal/vertical edges within and between CTUs DMVR [23] is used for merge candidates only when length of the motion vector is less than a pre-defined threshold. Disabling DMVR based on the length of the motion vector increases the DMVR parallelism.

\section{Conclusions}

The proposed VVC software player design generates real-time performance for four $4 \mathrm{~K}$ sequences i.e. CatRobot1, DaylightRoad2, Tango2 and FoodMarket4 at QP of 37. The proposed Multithreaded (MT) framework uses CTU level parallel processing techniques without compromising on the memory bandwidth.

The proposed method achieves 28 to 36 fps for CatRobot1, DaylightRoad2, Tango2 and FoodMarket4 sequences at QP: 32 to 37. 63 to $120 \mathrm{fps}$ achieved for all class B sequences at QP: 27 to 37. An overall average decoding time reduction of $88.97 \%$ is observed for the above mentioned ten sequences at QP values of 22, 27, 32, 37. The following average percentage reductions are observed for the individual decoding sub-tasks i.e.: MVCD: $70.17 \%$, Inter reconstruction: $88.25 \%$, Intra CU reconstruction: $68.94 \%$, Inverse Re-shaper: $86.79 \%$, ALF: $88.17 \%$, SAO: $72.28 \%$, DBF: $90.22 \%$.

\section{REFERENCES}

[1] High Efficiency Video Coding (HEVC), Rec. ITU-T H.265 and ISO/IEC 23008-2, January 2013 (and later editions).

[2] G. J. Sullivan, J.-R. Ohm, W.-J. Han, and T. Wiegand, "Overview of the High Efficiency Video Coding (HEVC) Standard", IEEE Trans. Circuits and Systems for Video Technology, Vol. 22, No. 12, pp. 1649-1668, December 2012.

[3] J. Chen, Y. Chen, M. Karczewicz, X. Li, H. Liu, L. Zhang, X. Zhao, "Coding tools investigation for next generation video coding”, ITU-T SG16 Doc. COM16-C806, February 2015.

[4] E. Alshina, A. Alshin, J. - H. Min, K. Choi, A. Saxena, M. Budagavi, "Known tools performance investigation for next generation video coding", VCEGAZ05, June 2015.

[5] A. Segall, V. Baroncini, J. Boyce, J. Chen, T. Suzuki, "Joint call for proposals on video compression with capability beyond HEVC", JVET-H1002, October 2017, Macau, China.

[6] B. Bross, K. Andersson, M. Blaser, V. Drugeon, S-H Kim, J. Lainema, J. Li, S. Liu, J. R. Ohm, G. J. Sullivan, "General Video Coding Technology in Responses to the Joint Call for Proposals on Video Compression with Capability beyond HEVC", IEEE Trans. Circuits and Systems for Video Technology, special issue October 2019

[7] J. Le. Tanou, M. Blestel, "Analysis of Emerging Video Codecs: Coding Tools, Compression Efficiency and Complexity", SMPTE Motion Imaging Journal, 128 (10), pp. 14-24, November 2019

[8] J. Chen, Y. Ye, S. H. Kim, “Algorithm description for Versatile Video Coding and Test Model 6(VTM 6)", JVET-O2002, July 2019.

[9] I. Ahmad, Y. He and M. L. Liou, "Video compression with parallel processing," Parallel Computing, 28 (7-8): 1039-1078, August 2002.

[10] DF De Souza, N. Roma, L. Sousa, "Co-operative CPU+GPU deblocking filter parallelization for high performance HEVC video codecs," IEEE 
International Conference on Acoustics, Speech and Signal Processing (ICASSP), pp. 4993-4997, May 2014.

[11] DF De Souza, A. llic, N. Roma, L. Sousa, "GPU assisted HEVC intra decoder," Journal of Real-Time Image Processing, vol. 12, Issue 2, pp. 531547, August 2016.

[12] DF De Souza, A. llic, L. Sousa, "HEVC in-loop GPU parallelization in embedded systems," International Conference on Embedded Computer System: Architectures, Modeling and Simulation (SAMOS XV), pp. 123-130, July 2015.

[13] W. Zhang, C. Guo, "Design and Implementation of Parallel Algorithms for Sample Adaptive Offset in HEVC Based on GPU", Sixth International Conference on Information Science and Technology, May 2016.

[14] Ittiam system private limited, "AHG16: Early Implementation of VVC software player and Demonstration on Mobile device", JVET-P0307, Geneva, CH, October 2019.

[15] A. M. Kotra, M. Raulet and O. Deforges, "Efficient Parallelization of Different HEVC Decoding Stages," 2013 Data Compression Conference, Snowbird, UT, pp. 502-502, March 2013.

[16] M. Alvarez-Mesa, C. C. Chi, B. Juurlink, V. George and T. Schierl, "Parallel video decoding in the emerging HEVC standard," 2012 IEEE International Conference on Acoustics, Speech and Signal Processing (ICASSP), Kyoto, pp. 1545-1548, March 2012.

[17] S. Gudumasu, Y. He, Y. Ye, Y. He, E-S. Ryu, J. Dong, X. Xiu, "Real-time SHVC software decoding with multi-threaded parallel processing", Proceedings of SPIE, Applications of Digital Image Processing, vol. 9217, September 2014

[18] M. Koziri et al., "Adaptive Tile Parallelization for Fast Video Encoding in HEVC," 2016 IEEE International Conference on Internet of Things (iThings) and IEEE Green Computing and Communications (GreenCom) and IEEE Cyber, Physical and Social Computing (CPSCom) and IEEE Smart Data (SmartData), Chengdu, pp. 738-743, December 2016

[19] V. Sze, A. P. Chandrakasan, M. Budagavi and Minhua Zhou, "Parallel CABAC for low power video coding," 2008 15th IEEE International Conference on Image Processing, San Diego, CA, pp. 2096-2099, October 2008.

[20] Y. Huang, et.al., "A VVC Proposal with Quaternary Tree plus BinaryTernary Tree Coding Block Structure and Advanced Coding Techniques," IEEE Transactions on Circuits and Systems for Video Technology, pp. 1-1, October 2019

[21] M. Wang et al., "Extended Quad-Tree Partitioning for Future Video Coding," 2019 Data Compression Conference (DCC), Snowbird, UT, USA pp. 300-309, May 2019.

[22] J. Chen, M. Karczewicz, Y-W. Huang, K. Choi, J-R. Ohm, G. J. Sullivan, "The Joint Exploration Model (JEM) for Video Compression with Capability beyond HEVC", IEEE Transactions on Circuits and Systems for Video Technology, pp. 1-1, October 2019.

[23] H. Gao, S. Esenlik, Z. Zhao, E. Steinbach, J. Chen, "Decoder Side Motion Vector Refinement for Versatile Video Coding", IEEE $21^{s t}$ International Workshop on Multimedia Signal Processing (MMSP), pp. 27-29, September 2019.

[24] L. Zhang, K. Zhang, H. Liu, H. C. Chuang, Y. Wang, J. Xu, P. Zhao, D. Hong, "History-Based Motion Vector Prediction in Versatile Video Coding", IEEE Data Compression Conference (DCC), pp. 43-52, March 2019.

[25] C.-C. Chen, X. Xiu, Y. He, Y. Ye, "Generalized bi-prediction method for future video coding", Proc. IEEE Picture Coding Symp. (PCS), pp. 1-5, December 2016

[26] L. Zhao et al., "Wide Angular Intra Prediction for Versatile Video Coding," 2019 Data Compression Conference (DCC), Snowbird, UT, USA, pp. 53-62, May 2019.

[27] Y. Chang et al., "Multiple Reference Line Coding for Most Probable Modes in Intra Prediction," 2019 Data Compression Conference (DCC), Snowbird, UT, USA, pp. 559-559, March 2019.

[28] A. Segall, J. Zhao and I. Yamamoto, "Parallel intra prediction for video coding," 28th Picture Coding Symposium, Nagoya, pp. 310-313, December 2010 .
[29] A. Kenneth, "Combined Intra Inter Prediction Coding Mode", Picture Coding Symposium ITU-T SG16/Q6 (VCEG) Doc. VCEGAD11, October 2006.

[30] Y. Wang, X. Guo, X. Fan, Y. Lu, D. Zhao and W. Gao, "Parallel In-Loop Filtering in HEVC Encoder on GPU," in IEEE Transactions on Consumer Electronics, vol. 64, no. 3, pp. 276-284, August 2018.

[31] A. Norkin et al., "HEVC Deblocking Filter," in IEEE Transactions on Circuits and Systems for Video Technology, vol. 22, no. 12, pp. 1746-1754, December 2012

[32] C. Fu et al., "Sample Adaptive Offset in the HEVC Standard," in IEEE Transactions on Circuits and Systems for Video Technology, vol. 22, no. 12, pp. 1755-1764, December 2012.

[33] VTM: the reference software for VVC development: https://vcgit.hhi.fraunhofer.de/ivet/VVCSoftware VTM

[34] F. Bossen, J. Boyce, K. Suehring, X. Li and V. Seregin, "JVET common test conditions and software reference configurations", JVET-L1010, Macao, CN, October 2018. 